\title{
A Exenteração Pélvica para o Câncer de Reto: Avaliação dos Fatores Prognósticos de Sobrevida em 27 Pacientes Operados
}

\author{
Pelvic Exenteration from Rectal Cancer: Evaluation of Survival Prognostic \\ Factors on 27 Resectable Cases
}

\author{
SERGIO RENATOPAIS COSTA ${ }^{1}$; ALEXANDRE CHARTUNI PEREIRATEIXEIRA ${ }^{2} ;$ RENATO ARIONI LUPINACCI $^{3}$
}

${ }^{1}$ Médico Assistente do Serviço de Cirurgia Geral e Oncocirurgia do Hospital do Servidor Público Estadual de São Paulo - "Francisco Morato de Oliveira"; ${ }^{2}$ Residente do Serviço de Cirurgia Geral e Oncocirurgia do Hospital do Servidor Público Estadual de São Paulo - "Francisco Morato de Oliveira"; ${ }^{3}$ Diretor do Serviço de Cirurgia Geral e Oncocirurgia do Hospital do Servidor Público Estadual de São Paulo - "Francisco Morato de Oliveira".

COSTA SRP; TEIXEIRA ACP; LUPINACCI RA. A Exenteração Pélvica para o Câncer de Reto: Avaliação dos Fatores Prognósticos de Sobrevida em 27 doentes Operados. Rev bras Coloproct, 2008;28(1): 007-018.

RESUMO: Objetivo - Identificar os fatores prognósticos de sobrevida dos pacientes submetidos à exenteração pélvica no tratamento curativo do câncer de reto (no Estádio T4 e na recidiva pélvica isolada). Métodos - Os dados completos de 27 pacientes submetidos a esse tipo de operação por adenocarcinoma de reto entre Janeiro de 1996 a Junho de 2006 foram avaliados. Foram estudados diversos fatores prognósticos epidemiológicos, cirúrgicos e histológicos por meio de análise multivariada. Resultados - A mortalidade pós-operatória foi de $7 \%(n=2)$ enquanto a morbidade global foi de $55 \%$ (n=15). A média de sobrevida global foi de 38 meses. A sobrevida global foi maior nos tumores T4 do que nas recidivas pélvicas (47 X 26 meses). Somente o comprometimento linfonodal $(\mathrm{N}+)$ foi fator prognóstico negativo na análise multivariada. Conclusão - A exenteração pélvica para o tratamento do câncer de reto apresenta alta morbidade e considerável mortalidade. Deve ser indicada nos tumores T4, principalmente quando não há disseminação linfonodal.

Descritores: Exenteração Pélvica, Câncer de Reto, Fatores Prognósticos, Morbidade, Mortalidade, Resultados do Tratamento.

\section{INTRODUÇÃOO}

O câncer de reto localmente avançado (T4) bem como a recidiva pélvica isolada representam situações clínicas desafiadoras na prática clínica diária. Ambas as condições condenam o doente a um sofri- mento atroz e duradouro, comprometendo seriamente tanto sua sobrevida em longo prazo quanto sua própria qualidade de vida. Apresentam frequiências variáveis nas diferentes séries descritas. Estima-se que entre 6 e $10 \%$ de todas as neoplasias retais se encontrem no estádio T4 no momento de seu diagnóstico (1-3). Por

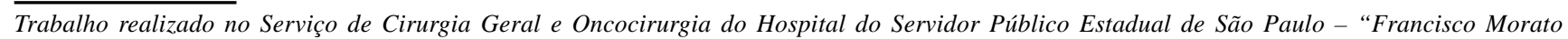
de Oliveira” - São Paulo - SP - Brasil. 
A Exenteração Pélvica para o Câncer de Reto: Avaliação dos Fatores Prognósticos de Sobrevida em 27 doentes Operados Sergio Renato Pais Costa e Cols.
Vol. 28 outro lado, as recidivas pélvicas após cirurgia potencialmente curativa têm variado de 4 a $50 \%$ (4-8). Somente a ressecção cirúrgica completa $(\mathrm{R} 0)$ e em monobloco, interessando tanto a neoplasia quanto os órgãos adjacentes comprometidos, pode oferecer real possibilidade de controle da doença em longo prazo nessa situação clínica $(1,2,4,7-10)$.

Descrita inicialmente por Brunschwig em 1948 para o tratamento da neoplasia de colo uterino recidivada, a exenteração pélvica foi considerada em seus primórdios procedimento paliativo com altas taxas de complicação e mortalidade $(9,11-16)$.

Atualmente, com o progresso da técnica operatória, das condições de suporte e dos cuidados pós-operatórios; essa operação tem sido indicada com âmbito curativo tanto para as neoplasias pélvicas localmente avançadas quanto para as recidivas pélvicas isoladas. Em que pese sua morbidade considerável, quando adequadamente indicada, como no caso de câncer de reto primário localmente avançado pode culminar com índices de sobrevida em cinco anos de até $60 \%(1,2,9,10)$.

Em contrapartida, para os doentes nessa mesma situação clínica não ressecados e submetidos a tratamento exclusivo com quimioterapia e radioterapia, a sobrevida em 5 anos não atinge $5 \%(5,6,8)$.

Panorama diferente é observado nas recidivas pélvicas isoladas, onde essa mesma abordagem terapêutica é raramente curativa. Cerca de $0 \%$ a $23 \%$ dos doentes mesmo após uma ressecção R0 alcançam 5 anos de sobrevida global $(2,11,15,16)$. Apesar dos resultados pouco expressivos, esse tipo de operação tem demonstrado controle duradouro dos sintomas nessa população levando assim a um benefício paliativo relevante $(5,8,15)$.

Poucos estudos, no que tange a avaliação de fatores prognósticos de sobrevida em longo prazo, têm sido conduzidos nessa rara situação clínica. Baseado nesse fato foi idealizado um estudo, cujo objetivo foi avaliar os fatores prognósticos de sobrevida em longo prazo em uma série de 27 pacientes com câncer de reto $\mathrm{T} 4$ e com recidiva pélvica isolada submetidos à exenteração pélvica no Serviço de Cirurgia Geral e Oncocirurgia do Hospital do Servidor Público Estadual de São Paulo, "Francisco Morato de Oliveira."

\section{MÉTODOS}

Foram analisados os prontuários de 62 doentes atendidos no Serviço de Cirurgia Geral e
Oncocirurgia do Hospital do Servidor Público Estadual de São Paulo, no período de 1996 a 2006. Quarenta e cinco doentes $(72,6 \%)$ apresentavam neoplasia de reto T4, enquanto dezessete $(27,4 \%)$ apresentavam recorrência pélvica após tratamento cirúrgico primário (recidiva pélvica). Dessa casuística inicial, vinte e cinco $(40,3 \%)$ foram excluídos de cirurgia radical devido a contra-indicações clínicas ou cirúrgicas (Tabela 1).

Destarte, trinta e sete pacientes foram inicialmente selecionados para exploração cirúrgica com o objetivo de avaliar a possibilidade de exenteração pélvica curativa. No entanto, seis foram excluídos de uma ressecção radical durante a avaliação intra-operatória. As causas foram: carcinomatose peritoneal $(n=3)$, metástases hepáticas $(n=2)$, metástases para linfonodos retroperitoneais $(n=1)$. Sendo assim, 31 exenterações pélvicas com intuito curativo foram realizadas no Serviço. Contudo, ainda foram excluídos para análise final quatro doentes, dois por apresentarem estadiamento T3 ao estudo histológico, e mais dois por perda de seguimento em longo prazo.

Os vinte e sete casos restantes que representaram a casuística final do presente estudo, foram divididos em dois grupos para comparação: Grupo 1 de 15 pacientes com neoplasia de reto T4 e Grupo 2 de 12 pacientes com recidiva pélvica exclusiva. As variáveis demográficas, clínicas e das neoplasias dos grupos estão representadas na Tabela 2.

Vinte e um pacientes $(77,7 \%)$ apresentavam sintomas incapacitantes. Sete pacientes apresentavam dois ou mais sintomas, enquanto que 6 pacientes não apresentavam qualquer sintoma. Todos os assintomáticos pertenciam ao Grupo 2. Essas características estão demonstradas na Tabela 3.

Todos os pacientes tiveram o diagnóstico de adenocarcinoma de reto confirmado por exame histológico. Diversas modalidades de estadiamento foram utilizadas no pré-operatório destes pacientes, incluindo exame físi-

Tabela 1 - Contra-indicações clínicas e cirúrgicas a exenteração $(n=25)$.

\begin{tabular}{lrc}
\hline Contra-indicação & No. & $(\boldsymbol{\%})$ \\
\hline Má Condição clínica & 9 & $(36)$ \\
Doença metastática à distância & 9 & $(36)$ \\
Envolvimento ósseo & 3 & $(12)$ \\
Envolvimento ureteral bilateral & 2 & $(8)$ \\
Envolvimento de artéria ilíaca externa & 1 & $(4)$ \\
\hline
\end{tabular}


Tabela 2 - Características epidemiológicas dos doentes $(n=27)$.

\begin{tabular}{|c|c|c|c|c|c|}
\hline & Grupo & 1 No. $(\%)$ & Grupo 2 No. (\%) & $P$ & Total No. (\%) \\
\hline \multicolumn{6}{|l|}{$\overline{\text { Sexo }}$} \\
\hline Masculino & 8 & $(53,3)$ & $6 \quad(50)$ & $n s$ & $14(51,9)$ \\
\hline Feminino & 7 & $(46,7)$ & $6 \quad(50)$ & $n s$ & $13(48,1)$ \\
\hline Média etária (anos) & 65,9 & $(36-77)$ & $58,7(45-75)$ & 0,017 & $62,7(36-77)$ \\
\hline \multicolumn{6}{|l|}{ Raça } \\
\hline Negra & 4 & $(26,7)$ & $4 \quad(33,3)$ & $n s$ & $8 \quad(29,6)$ \\
\hline Branca & 11 & $(73,3)$ & $7 \quad(58,3)$ & $n s$ & $18 \quad(66,7)$ \\
\hline Amarela & 0 & $(0)$ & $(8,3)$ & $n s$ & $1 \quad(3,7)$ \\
\hline \multicolumn{6}{|l|}{ Risco Anestésico (ASA) } \\
\hline ASA I & 9 & $(60)$ & $9 \quad(75)$ & $n s$ & $18 \quad(66,7)$ \\
\hline ASA II & 5 & $(33,3)$ & $2 \quad(16,7)$ & $n s$ & $7 \quad(25,9)$ \\
\hline ASA III & 1 & $(6,7)$ & $(8,3)$ & $n s$ & $2 \quad(7,4)$ \\
\hline \multicolumn{6}{|l|}{ Altura do tumor } \\
\hline Superior & 2 & $(13,3)$ & (0) & $n s$ & $2 \quad(7,4)$ \\
\hline Médio & 7 & $(46,7)$ & $(91,7)$ & $n s$ & $18 \quad(66,7)$ \\
\hline Inferior & 6 & $(40)$ & $(8,3)$ & $n s$ & $7 \quad(25,9)$ \\
\hline CEA pré-operatório médio (ng/mL) & 99,74 & $(1,5-456)$ & $160,86(2-531)$ & $n s$ & $126,9(1,5-531)$ \\
\hline CA 19.9 pré-operatório médio $(\mathrm{ng} / \mathrm{mL})$ & 108,26 & $(5-436)$ & $122,08(5-678)$ & $n s$ & $114,4(5-678)$ \\
\hline Tratamento neoadjuvante & 11 & $(73,3)$ & $4 \quad(33,3)$ & $n s$ & $15(55,5)$ \\
\hline \multicolumn{6}{|l|}{ Tratamento adjuvante } \\
\hline Quimioterapia & 3 & $(20)$ & $5 \quad(41,6)$ & $n s$ & $8(29,6)$ \\
\hline Radioterapia + Quimioterapia & 1 & $(6,7)$ & $(0)$ & & $1 \quad(3,7)$ \\
\hline \multicolumn{6}{|l|}{ Tipo de cirurgia } \\
\hline Exenteração pélvica total (EPT) & 9 & $(60)$ & $(66,7)$ & $n s$ & $17 \quad(62,9)$ \\
\hline Exenteração pélvica posterior (EPP) & 3 & $(20)$ & $(8,3)$ & $n s$ & $4(14,8)$ \\
\hline EPT com sacrectomia & 1 & $(6,7)$ & (25) & $n s$ & $4 \quad(14,8)$ \\
\hline EPP com cistectomia parcial & 2 & $(13,3)$ & $(0)$ & $n s$ & $2 \quad(7,4)$ \\
\hline \multicolumn{6}{|l|}{ Reconstruções urinárias } \\
\hline Ureteroileostomias (Bricker) & 10 & $(83,3)$ & $11 \quad(100)$ & $n s$ & $21 \quad(91,3)$ \\
\hline Ureteroneocistostomias (Boari) & 2 & $(16,7)$ & $0 \quad(0)$ & $n s$ & $2(8,7)$ \\
\hline
\end{tabular}

ASA - classificação da American Society of Anesthesiologysts; CEA - Antígeno Cárcino-Embrionário;

Tratamento neoadjuvante, radio-quimioterapia; $n s$ - não significante.

co, raio-x de tórax, tomografia (TC) de abdome e pelve, ressonância nuclear magnética (RNM) de pelve, ultrasonografia endoanal. A tomografia com emissão de pósitrons (PET-SCAN) foi realizada de acordo com a necessidade do caso. Foram dosados em todos doentes o antígeno carcinoembrionário e o CA 19,9 séricos. A sua distribuição esta demonstrada na tabela 2.

No Grupo 2, em relação às cirurgias prévias realizadas para o tratamento dos doentes, foi observada a seguinte distribuição: ressecção anterior baixa com anastomose mecânica colo-anal $(n=9)$, anastomose
Tabela 3 - Sinais e sintomas dos doentes $(n=27)$.

\begin{tabular}{lrr}
\hline & No. & (\%) \\
\hline Dor pélvica & 17 & $(62,9)$ \\
Sangramento retal & 9 & $(33,3)$ \\
Infecção urinária recorrente & 3 & $(11,1)$ \\
Fístula reto-vaginal & 1 & $(3,7)$ \\
Fístula reto-vesical & 1 & $(3,7)$ \\
Dois ou mais sintomas & 7 & $(25,9)$ \\
Assintomáticos & 6 & $(22,2)$ \\
\hline
\end{tabular}


A Exenteração Pélvica para o Câncer de Reto: Avaliação dos Fatores Prognósticos de Sobrevida em 27 doentes Operados Sergio Renato Pais Costa e Cols. manual colo-retal baixa $(n=1)$, ressecção anterior baixa com colostomia terminal - Cirurgia Tipo Hartman $(\mathrm{n}=2)$. Em relação ao estadiamento histológico (TNM) pós-operatório houve a seguinte distribuição: T3N0M0 $(\mathrm{n}=2), \mathrm{T} 3 \mathrm{~N} 1 \mathrm{M} 0(\mathrm{n}=8)$ e T3N2M0 $(\mathrm{n}=2)$.

Todas as cirurgias foram realizadas por uma única equipe cirúrgica especializada. O cirurgião responsável por cada procedimento apresentava treinamento extensivo em cirurgia oncológica pélvica, com pelo menos vinte ressecções multiviscerais pélvicas prévias durante a curva de aprendizado.

Foram realizadas no total: 17 exenterações pélvicas totais (EPT), 4 exenterações pélvicas posteriores (EPP), 4 EPTs com sacrectomia e 2 EPP com cistectomia parcial e ureterectomia. As reconstruções urinárias utilizadas foram: ureteroileostomias a Bricker $(\mathrm{N}=21)$ e ureteroneocistostomias a Boari $(\mathrm{N}=2)$. Em seis pacientes foram realizadas cirurgias com preservação de esfíncter (anastomose colo-anal ou colo-retal).

Quinze doentes receberam tratamento radio e quimioterápico neo-adjuvante com os objetivos de redução do volume tumoral, melhoria da ressecabilidade e diminuição do estadiamento clínico-radiológico (downstaging). Onze pertenciam ao Grupo 1, enquanto quatro pertenciam ao Grupo 2. Oito pacientes receberam quimioterapia adjuvante e apenas um paciente recebeu radio-quimioterapia pós-operatória. Dezoito pacientes não foram submetidos a qualquer tipo de terapia adjuvante.

Todos os pacientes foram acompanhados no pós-operatório conjuntamente pelo Serviço de Cirurgia Geral e Oncocirurgia e pelo Serviço de Oncologia Clínica do Hospital do Servidor Público Estadual de São Paulo. Exame físico, dosagem de antígeno carcinoembrionário (CEA), CA19.9, RX de tórax, TC de abdome e pelve foram realizados a cada 3 meses até 2 anos após a cirurgia. Após 2 anos, os pacientes foram avaliados a cada 6 meses. Uma colonoscopia anual foi realizada em cada paciente. O PET-SCAN foi realizado na dúvida quanto à recidiva tumoral nos últimos cinco doentes dessa série. Toda recidiva foi confirmada por exame de imagem (TC) e por avaliação histológica.

Foram analisadas retrospectivamente as seguintes variáveis: idade, sexo, cor, tipo do tumor (primário ou recidivado), grau de diferenciação, altura em relação à borda anal, níveis séricos de marcadores tumorais, tempo operatório, volume de sangramento intra-operatório, necessidade de transfusão, volume de transfusão, tempo de internação pós-operatório, morbidade, tratamento multimodal, comprometimento linfonodal e mortalidade. Morbidade foi definida como qualquer complicação pós-operatória ocorrida durante a internação. A mortalidade foi definida como óbito durante a internação hospitalar.

O tempo de sobrevida foi calculado a partir da data da ressecção da lesão até a última consulta durante o acompanhamento, ou até o óbito. A análise estatística foi realizada utilizando-se o programa EpiInfo 3.4.1, para Windows. Os índices de sobrevida cumulativa, sobrevida livre de doença foram calculados de acordo com o método de Kaplan-Meier, e os fatores prognósticos foram analisados com o uso do Log-rank test. Foram realizadas análises uni e multivariada com o modelo de regressão logística de Cox. Foi considerado estatisticamente significante um $\mathrm{p}<0,05$.

\section{RESULTADOS}

No que tange as características epidemiológicas dos doentes, não houve diferença estatisticamente significante em relação as diferentes variáveis estudadas, exceto a idade cuja média etária foi superior no Grupo 1 (Tabela 1). Em relação aos achados cirúrgicos, não houve diferença estatisticamente significante entre os grupos nas diversas variáveis estudadas, exceto o número médio de transfusões em unidades que foi estatisticamente maior no Grupo 2 (Tabela 4).

Tabela 4 - Características das cirurgias $(n=27)$.

\begin{tabular}{lccccccc}
\hline & Grupo 1 No. (\%) & Grupo 2 No. (\%) & P & \multicolumn{2}{c}{ Total No. (\%) } \\
\hline Tempo operatório médio (min) & $420,33(280-485)$ & 410,25 & $(228-550)$ & 0,730 & $415,85(228-550)$ \\
Sangramento intra-operatório (ml) & 1947 & $(600-4800)$ & 2392 & $(600-5000)$ & 0,313 & 2144 & $(600-5000)$ \\
Necessidade de transfusão & 13 & $(86,7)$ & 11 & $(91,7)$ & 0,823 & 24 & $(88,9)$ \\
Número médio de transfusões (unidades) & 5,46 & $(3-8)$ & 8,9 & $(5-14)$ & 0,015 & 7,04 & $(3-14)$ \\
Tempo médio de internação (dias) & 19 & $(7-28)$ & 21,75 & $(9-34)$ & 0,301 & 20,22 & $(7-34)$ \\
\hline
\end{tabular}


A morbidade global da presente série foi de $55,5 \%$. Quinze doentes apresentaram pelo menos uma complicação (Tabela 5). A proporção de complicações foi similar entre os grupos, e não houve diferença estatisticamente significante entre os mesmos em relação ao número de complicações (Grupo 1- 53,3\% e Grupo 2- 58,33\%). Em três pacientes houve necessidade de reintervenção cirúrgica devido a complicações. Todos três apresentaram abscessos intra-cavitários, e necessitaram de laparotomia para sua drenagem. Dois deles pertenciam ao Grupo 2.

A mortalidade global da série foi de $7,4 \%$. A mortalidade foi similar entre os dois grupos $(6,6 \% \mathrm{X}$ $8,3 \%$ ), e não houve diferença estatisticamente significativa em relação a essa variável. Um paciente em cada grupo faleceu durante a internação. No Grupo 1, um doente submetido a exenteração com ressecção óssea, faleceu devido à embolia pulmonar mesmo em uso de profilaxia de trombose venosa profunda. No Grupo 2, um doente evoluiu com abscesso pélvico, peritonite e choque séptico, culminando com o óbito.

Em vinte e quatro pacientes $(88,8 \%)$, as margens de ressecção foram livres de comprometimento neoplásico (Ressecção R0). Os três pacientes restantes $(11,2 \%)$, todos pertencentes ao Grupo 2, apresentaram doença residual microscópica (Ressecção R1). Apesar dessa diferença, ela não foi estatisticamente significante entre os grupos. Os órgãos invadidos pela neoplasia retal foram: bexiga $(n=12)$, próstata $(n=7)$, útero $(n=7)$, vesículas seminais $(n=3)$, vagina $(n=3)$, ureter $(n=2)$ e sacro $(n=1)$. O grau histológico e de diferenciação dos tumores ressecados também foi estudado: oito pacientes $(29,7 \%)$ apresentaram tumores bem diferenciados, nove pacientes $(33,3 \%)$ tiveram tumores moderadamente diferenciados, e dez pacientes $(37 \%)$ tiveram neoplasias pouco diferenciadas. Proporcionalmente, o Grupo 2 apresentou mais pacientes com tumores moderadamente ou pouco diferenciados do que o Grupo 1 (83,3\% x 60\%), no entanto não houve diferença estatisticamente significante entre os grupos.

O número de linfonodos dissecados nos pacientes do Grupo 1 variou de 18 a 43 linfonodos, com mediana de 28 linfonodos. No Grupo 2 a mediana foi de 24 linfonodos, variando de 17 a 39 linfonodos. Não houve diferença estatisticamente significante em relação ao número de linfonodos dissecados entre os grupos $(P=0,1101)$. Nove $(33,3 \%)$ apresentaram linfonodos comprometidos, sendo quatro pertencentes ao Grupo 1 $(26,7 \%)$ e 5 pertencentes ao Grupo $2(41,7 \%)$. As características histológicas e de acompanhamento dos pacientes estão expostas na Tabela 6.

A remissão dos sintomas pré-operatórios foi atingida em $93,3 \%$ dos pacientes do Grupo 1, enquanto que no Grupo 2 foi atingida em apenas 50\% dos indivíduos previamente sintomáticos.

O tempo de acompanhamento pós-operatório variou de 13 a 80 meses, com média de 37,72 meses. Treze pacientes $(48,1 \%)$ apresentaram recidiva tumoral local ou à distância durante o período de acompanha-

Tabela 5 - Taxas de morbidade e mortalidade $(n=27)$.

\begin{tabular}{|c|c|c|c|}
\hline & Grupo 1 No. (\%) & Grupo 2 No. (\%) & Total No. $(\%)$ \\
\hline Complicações pós-operatórias (p=ns) & $8 \quad(53,3)$ & $7 \quad(58,3)$ & $15(55,5)$ \\
\hline Abscesso / Peritonite & $(6,66)$ & 3 (25) & $4(14,8)$ \\
\hline Infeccão do trato urinário & $2 \quad(13,3)$ & $(8,3)$ & $3(11,1)$ \\
\hline Pneumonia & $1 \quad(6,6)$ & $(8,3)$ & $2(7,4)$ \\
\hline Fístula retovesical & $(6,6)$ & (0) & $1 \quad(3,7)$ \\
\hline Fístula urinária & $(0)$ & $(8,3)$ & $1 \quad(3,7)$ \\
\hline Insuficiência renal aguda & $(6,6)$ & (0) & $1 \quad(3,7)$ \\
\hline Tromboembolismo pulmonar & $(6,6)$ & (0) & $1 \quad(3,7)$ \\
\hline Trombose venosa profunda & $(0)$ & $(8,3)$ & $1 \quad(3,7)$ \\
\hline Íleo prolongado & $(6,6)$ & $(0)$ & $1 \quad(3,7)$ \\
\hline Necessidade de reoperação $(\mathrm{p}=\mathrm{ns})$ & $(6,6)$ & $2(16,6)$ & $3(11,1)$ \\
\hline Mortalidade $(\mathrm{p}=\mathrm{ns})$ & $(6,6)$ & $(8,3)$ & $2(7,4)$ \\
\hline
\end{tabular}

$n s-n \tilde{a} o$ significante. 
Tabela 6 - Características histológicas e de seguimento $(n=27)$.

\begin{tabular}{|c|c|c|c|c|c|c|c|}
\hline \multirow{2}{*}{ Órgãos e estruturas invadidos } & \multicolumn{2}{|c|}{ Grupo 1 No. (\%) } & \multicolumn{2}{|c|}{ Grupo 2 No. (\%) } & \multirow[t]{2}{*}{$P$} & \multicolumn{2}{|c|}{ Total No. (\%) } \\
\hline & & & & & & & \\
\hline Bexiga & 11 & $(73,3)$ & 1 & $(8,3)$ & & 12 & $(44,4)$ \\
\hline Próstata & 1 & $(6,6)$ & 6 & $(50)$ & & 7 & $(25,9)$ \\
\hline Útero & 3 & (20) & 4 & $(33,3)$ & & 7 & $(25,9)$ \\
\hline Vesículas seminais & 1 & $(6,6)$ & 2 & $(16,6)$ & & 3 & $(11,1)$ \\
\hline Vagina & 0 & (0) & 3 & $(25)$ & & 3 & $(11,1)$ \\
\hline Ureter & 0 & (0) & 2 & $(16,6)$ & & 2 & $(7,4)$ \\
\hline Sacro & 1 & $(6,6)$ & 3 & (25) & & 4 & $(14,8)$ \\
\hline \multicolumn{8}{|l|}{ Ressecção } \\
\hline R0 & 15 & (100) & 9 & $(75)$ & $n s$ & 24 & $(88,8)$ \\
\hline $\mathrm{R} 1$ & 0 & $(0)$ & 3 & (25) & $n s$ & 3 & $(11,2)$ \\
\hline \multicolumn{8}{|l|}{ Grau de diferenciação da neoplasia } \\
\hline Bem diferenciado & 6 & (40) & 2 & $(16,7)$ & $n s$ & 8 & $(29,7)$ \\
\hline Moderadamente diferenciado & 5 & $(33,3)$ & 4 & $(33,3)$ & $n s$ & 9 & $(33,3)$ \\
\hline Pouco diferenciado & 4 & $(26,7)$ & 6 & $(50)$ & $n s$ & 10 & (37) \\
\hline \multicolumn{8}{|l|}{ Linfonodos } \\
\hline Média de linfonodos dissecados & 28,9 & $3(18-43)$ & 24,5 & $(17-39)$ & $n s$ & 26,96 & $(17-43)$ \\
\hline Pacientes com metástases linfonodais & 4 & $(26,7)$ & 5 & $(41,6)$ & $n s$ & 9 & $(33,3)$ \\
\hline Média de metástases linfonodais* & 2,7 & $5(1-4)$ & 3,4 & $(2-5)$ & $n s$ & 3,11 & $(1-5)$ \\
\hline Remissão completa dos sintomasI & 14 & $(93,3)$ & 3 & $(50)$ & & 17 & $(80,9)$ \\
\hline Recidiva tumoral & 5 & $(33,3)$ & 8 & $(66,7)$ & $n s$ & 13 & $(48,1)$ \\
\hline Pélvica & 0 & (0) & 5 & $(41,6)$ & & 5 & $(18,5)$ \\
\hline Hepática & 2 & $(13,3)$ & 2 & $(16,6)$ & & 4 & $(14,8)$ \\
\hline Peritonial & 2 & $(13,3)$ & 0 & (0) & & 2 & $(7,4)$ \\
\hline Pulmonar & 1 & $(6,6)$ & 0 & (0) & & 1 & $(3,7)$ \\
\hline Múltiplas & 0 & $(0)$ & 1 & $(8,3)$ & & 1 & $(3,7)$ \\
\hline \multicolumn{8}{|l|}{ Sobrevida (meses) } \\
\hline Sobrevida média & 47 & $(13-80)$ & 26 & $(13-48)$ & 0,0076 & 37,72 & $(13-80)$ \\
\hline Sobrevida estimada em 3 anos & & (69) & & (34) & $n s$ & & $(53)$ \\
\hline Sobrevida estimada em 5 anos & & $(61)$ & & $(0)$ & $n s$ & & (34) \\
\hline Pacientes com metástases linfonodais & 24,3 & $3(20-28)$ & 22,25 & $(17-30)$ & $n s$ & 23 & $(17-30)$ \\
\hline Pacientes sem metástases linfonodais & 53,0 & $9(13-80)$ & 28,14 & $(13-48)$ & 0,0235 & 43 & $(13-80)$ \\
\hline
\end{tabular}

* Nos pacientes com metástases linfonodais

I Nos pacientes previamente sintomáticos, NS - não significante.

mento. Cinco pertenciam ao Grupo $1(33,3 \%)$, e 8 pertenciam ao Grupo $2(66,7 \%)$. Os locais de recidiva foram: pelve $(n=5)$, fígado $(n=4)$, peritônio $(n=2)$ e pulmão $(n=1)$. Em um paciente, pertencente ao Grupo 2, foi observado recidiva múltipla. A sobrevida livre de doença nos que apresentaram recorrência durante o seguimento variou de 7 a 30 meses, com média de 15 meses. Nos pacientes do Grupo 1 que apresentaram recidiva, foi observado uma maior sobrevida livre de doença, com média de 20 meses, em comparação aos pacientes do Grupo 2 com recidiva (média de 12 meses), contudo sem diferença estatisticamente significante $(\mathrm{P}=0,0926)$. Apenas os pacientes do Grupo 2 apresentaram recidivas locais (5 pacientes, $41,6 \%)$.

A sobrevida média global dos 27 pacientes foi de 37,72 meses (13-80 meses). Quando realizada a comparação entre grupos, observou-se que os pacien- 
tes do Grupo 1 apresentaram uma sobrevida média significativamente maior. A sobrevida média no Grupo 1 foi de 47 meses de sobrevida (13 a 80 meses), enquanto no Grupo 2 foi de 26 meses (13 a 48 meses). Houve diferença estatisticamente significante na sobrevida média entre os grupos $(\mathrm{P}=0,0076)$. As curvas de probabilidade de sobrevida podem ser vistas na Figura 1.

A sobrevida estimada (Kaplan-Meier) aos 3 anos foi de $69 \%$ para o Grupo 1, e de $34 \%$ para o Grupo 2. Em cinco anos a sobrevida estimada no Grupo 1 foi de $61 \%$, enquanto que no Grupo 2 foi de $0 \%$. Em que pese essa diferença, ela não foi estatisticamente significante $(\mathrm{P}=0,05)$.

Houve diferença na sobrevida dos pacientes de acordo com o grau de diferenciação tumoral. A sobrevida em longo prazo dos doentes com neoplasias bem diferenciadas, moderadamente diferenciadas e pouco diferenciadas foi respectivamente: $100 \%, 53 \%$ e $0 \%(\mathrm{P}=0,0001)$. Esses dados estão demonstrados na Figura 2. Quando comparada a radicalidade da cirurgia (R0 X R1), também foi encontrada maior sobrevida nos pacientes submetidos à ressecção R0 quando comparados com ressecção R1 (52\% e 0\% de sobrevida em 5 anos). Houve diferença estatisticamente significante em relação a essa variável $(\mathrm{P}=0,0001)$. Esses dados estão demonstrados na Figura 3.

Todos os pacientes que apresentaram linfonodos comprometidos na avaliação histológica, desenvolveram algum tipo de recidiva e evoluíram a óbito pela doença. Os pacientes com linfonodos

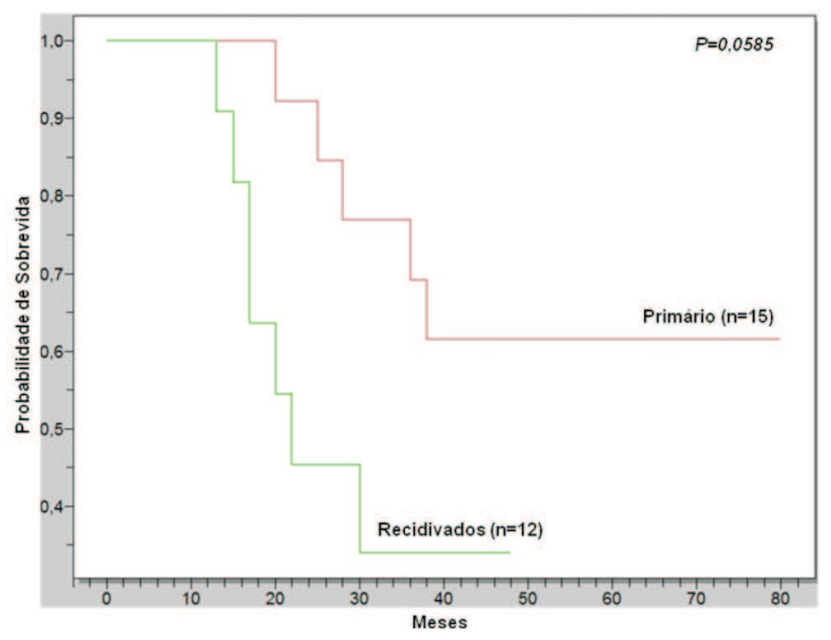

Figura 1 - Probabilidade de sobrevida entre grupos. comprometidos apresentaram sobrevida média de 23 meses (17-30 meses), enquanto que os pacientes com linfonodos livres de comprometimento neoplásico tiveram sobrevida variando entre 13 e 80 meses, com média de 43 meses. Sessenta e nove por cento dos pacientes com linfonodos negativos alcançaram 5 anos de sobrevida, contrastando com nenhum dos pacientes com linfonodos comprometidos $(\mathrm{P}=0,0005)$. As curvas de probabilidade de sobrevida estão na Figura 4.

No Grupo 1, a média de sobrevida dos pacientes com linfonodos comprometidos foi de 24,33 meses e nos pacientes com linfonodos livres foi de 53,09 meses $(\mathrm{P}=0,03)$. No Grupo 2, as médias de sobrevida foram de 22,25 e 28,14 meses para os pacientes com linfonodos positivos e negativos, respectivamente, não atingindo diferença estatística $(\mathrm{P}=0,4531)$.

Foram avaliadas as variáveis clínicas, laboratoriais e histológicas que pudessem ser preditores relevantes de sobrevida em longo prazo nos 27 pacientes submetidos à exenteração pélvica. Ao se realizar a análise univariada dos fatores prognósticos (Tabela 7), as variáveis associadas a um prognóstico adverso foram: sexo masculino, presença de linfonodos comprometidos pela neoplasia e o tipo de tumor (recidiva pélvica). No entanto, ao selecionar esses fatores para análise multivariada (Tabela 8), o único que manteve correlação estatisticamente significante com a sobrevida, considerado fator prognóstico negativo independente foi à presença de linfonodo comprometido $(\mathrm{P}=0,0167)$.

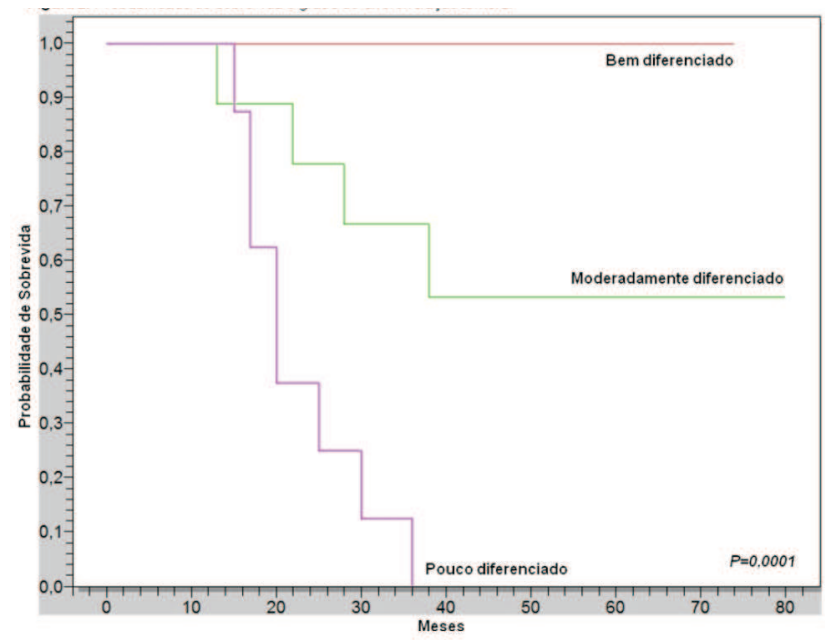

Figura 2 - Probabilidade de sobrevida e graus de diferenciação tumoral. 


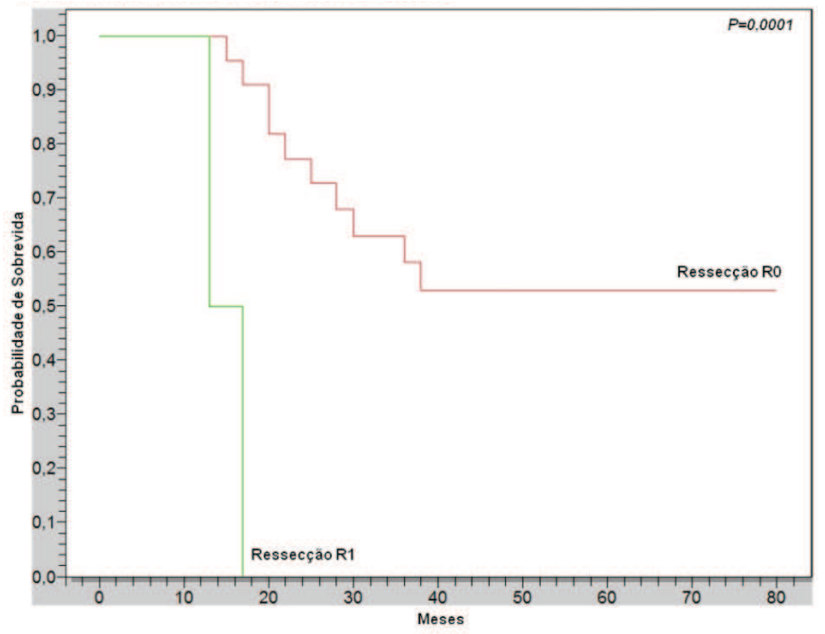

Figura 3 - Probabilidade de sobrevida e margens de ressecção.

\section{DISCUSSÃO}

O tratamento do câncer de reto T4 ou da recidiva pélvica isolada é um problema de difícil solução técnica. Cerca de 6 a $10 \%$ dos pacientes com neoplasia retal primária apresentam tumores avançados, com envolvimento de órgãos e estruturas adjacentes. Além disso, após ressecção retal curativa, de 4 a 50\% dos pacientes evoluem com recidiva local do tumor $(1,2)$,

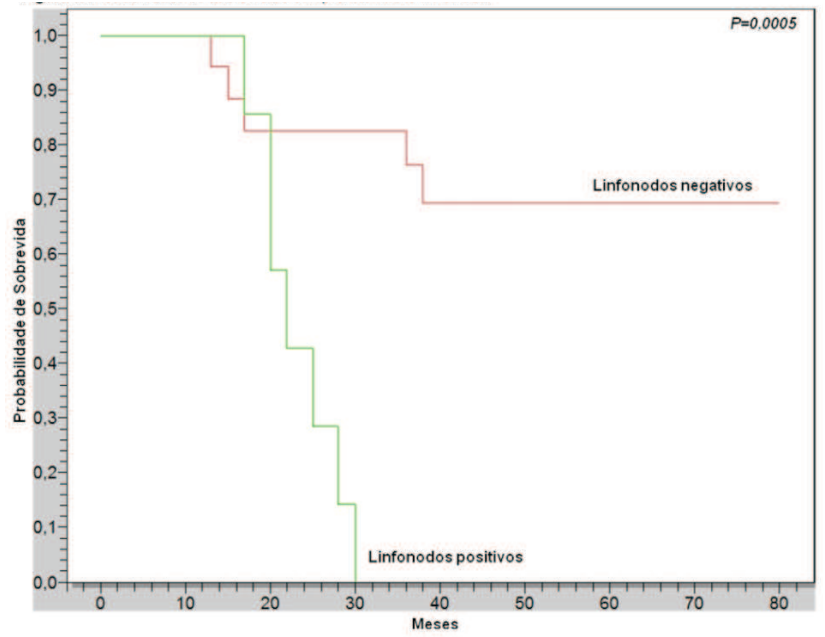

Figura 4 - Probabilidade de sobrevida e comprometimento linfonodal.

apesar dos avanços no tratamento como a radioquimioterapia neo-adjuvante e a excisão total do mesorreto $(7,10,18,19)$. Esses pacientes apresentam em geral sintomatologia exuberante, o que os condena a um sofrimento severo. Sintomas como dor pélvica, infecções urinárias de repetição, sangramento retal e fístulas tanto reto-vaginais quanto reto-vesicais são comumente observados nessas situações além de uma curta expectativa de vida.

Tabela 7 - Análise univariada de fatores prognósticos.

\begin{tabular}{lccc}
\hline & Hazard Ratio $(\mathbf{9 5 \%}$ CI) & P \\
\hline Idade (anos) & 0,97 & $(0,90-1,05)$ & 0,56 \\
Sexo (feminino/masculino) & 0,33 & $(0,08-1,23)$ & 0,0998 \\
ASA (II/I) & 1,03 & $(0,27-3,94)$ & 0,9547 \\
ASA (III/I) & 1,32 & $(0,16-10,64)$ & 0,7906 \\
Tratamento neoadjuvante (sim/não) & 0,86 & $(0,27-2,68)$ & 0,7983 \\
Radicalidade (R1/R0) & 2,80 & $(0,22-35,28)$ & 0,42 \\
Metástases linfonodais (sim/não) & $8,33 \quad(2,03-34,00)$ & 0,0032 \\
Tipo do tumor (recidivado/primário) & 2,91 & $(0,90-9,36)$ & 0,0726 \\
\hline
\end{tabular}

$P<0,1$ - incluídos na análise multivariada

CI- intervalo de confiança

Tabela 8 - Análise multivariada de fatores prognósticos.

\begin{tabular}{lccc}
\hline & Hazard Ratio (95\% CI) & P \\
\hline Sexo (masculino/feminino) & 0,47 & $(0,12-1,83)$ & 0,277 \\
Metástases linfonodais (sim/não) & 5,66 & $(1,36-23,39)$ & 0,0167 \\
Tipo do tumor (recidivado/primário) & 2,22 & $(0,67-7,31)$ & 0,1868 \\
\hline
\end{tabular}


A Exenteração Pélvica para o Câncer de Reto: Avaliação dos Fatores Prognósticos de Sobrevida em 27 doentes Operados Sergio Renato Pais Costa e Cols.
Em que pese à alta morbidade da exenteração pélvica, essa é a única oportunidade de controle da doença em longo prazo, haja vista que os demais tratamentos têm apresentado resultados desalentadores (1-19).

Nos primórdios da descrição desta operação, os resultados eram desapontadores com altos índices de morbi-mortalidade e sobrevida pífia. No entanto, com o refinamento da técnica cirúrgica, melhor avaliação radiológica pré-operatória, evolução dos cuidados anestésicos e de pós-operatório houve uma crescente melhora de seus resultados. Tudo isso tem proporcionado uma diminuição significativa da mortalidade pósoperatória nas últimas décadas (2). Em estudos mais recentes, os índices têm variado de 0 e $20 \%$ (1-16).

Embora o presente estudo não tenha sido realizado em um hospital especializado em câncer, a mortalidade global de 7,4\% se encontra dentro dos números mais recentemente descritos na literatura (2). Acreditamos que a melhora nas taxas de mortalidade, se deve às inovações técnicas cirúrgico-anestésicas aliadas à melhor seleção dos pacientes e paralelamente ao melhor cuidado intensivo pós-operatório.

Em contrapartida, a morbidade ainda permanece alta com índices que tem variado de 50 a $67,6 \%$ na literatura $(1,2)$. A morbidade do presente estudo de $55 \%$ é similar à descrita na literatura. As maiorias das complicações observadas nessa série foram consideradas como menores, não se correlacionando na maioria dos casos com períodos de internação prolongados.

O número de reintervenções por complicação infecciosa (abscesso-peritonite) no presente estudo não foi desprezível (10\%), contudo vale recordar que esses pacientes foram submetidos à radioterapia pré-operatória, e também foram os primeiros casos dessa série quando a drenagem percutânea de abscessos era indisponível em nosso serviço. Têm sido associadas complicações infecciosas com mortalidade pós-operatória, no entanto somente um desses doentes acabou por falecer devido a esse tipo de complicação. Paralelamente, é importante lembrar que a presença de complicações maiores onde uma reintervenção é necessária pode acarretar um retardo no tratamento adjuvante quando necessário o que pode piorar o prognóstico final.

Em que pese à ascensão do tratamento neoadjuvante (radioterapia e quimioterapia combinadas) para o tratamento do câncer de reto localmente avançado $(\mathrm{T} 3-4 / \mathrm{N}+)$ como tendência na literatura $(18,19)$ com os objetivos de diminuição da recidiva pélvica, maior conservação esfincteriana e de downstaging; nem todos os casos são passíveis dessa abordagem. Pacientes previamente irradiados, multiplamente operados ou com fístula tanto reto-vaginal quanto retovesical, por exemplo, não são candidatos a essa estratégia, e somente lhes resta a exenteração como forma curativa de tratamento. Embora os índices de resposta ao tratamento neo-adjuvante, principalmente com novos agentes quimioterápicos e radioterapia conformacional apresentem resultados cada vez mais animadores, nem todos pacientes responderão ou tolerarão essa abordagem terapêutica apenas lhes restando também como alternativa terapêutica curativa a exenteração pélvica.

Em contrapartida, pacientes com tumores localmente avançados T4 tratados exclusivamente com radio-quimioterapia apresentam sobrevida curta. Estima-se que menos que $5 \%$ desses indivíduos alcancem 5 anos de sobrevida $(5,6)$.

Os resultados com essa técnica cirúrgica para os tumores T4 são conhecidos de longa data. Essa formidável operação, quando adequadamente indicada em indivíduos selecionados, pode levar a um prolongamento da sobrevida ou até mesmo a cura. Em estudos mais recentes, a sobrevida em cinco anos dos doentes com tumores $\mathrm{T} 4$ submetidos a esse procedimento tem se situado em torno de até $70 \%(1,2,9,10)$. Em nosso estudo, em que pese sua pequena casuística observamos resultados similares (sobrevida estimada $61 \%$ em 5 anos), principalmente naqueles sem comprometimento linfonodal, e que foram a maioria dos casos resseca$\operatorname{dos}(73 \%)$.

Por outro lado, é sabido que os resultados em termos de sobrevida em longo prazo após a exenteração nas recidivas pélvicas isoladas são duvidosos quando comparados aos resultados amplamente favoráveis observados naqueles doentes com doença primária localmente avançada (2). Fato este que ficou confirmado no presente estudo, haja vista que nenhum dos pacientes do grupo de recidiva pélvica atingiu 5 anos de sobrevida. No entanto, a comparação desses dados entre as várias instituições é difícil, provavelmente, devido as suas diferentes indicações dentro de cada instituição (2).

Bedrosian et al. (7) ao estudarem pacientes submetidos à cirurgia para neoplasias retais com recidiva pélvica isolada, encontraram sobrevida em 5 anos 
A Exenteração Pélvica para o Câncer de Reto: Avaliação dos Fatores Prognósticos de Sobrevida em 27 doentes Operados Sergio Renato Pais Costa e Cols. de $36 \%$. Entretanto, esta amostra de pacientes envolve diversas modalidades de cirurgia, incluindo procedimentos menos agressivos do que a exenteração, além de neoplasias retais menos avançadas (<T3). Em linhas gerais, em estudos mais recentes os resultados têm sido pífios, e têm variado de 0 até $23 \%$ de sobrevida em cinco anos $(2,11,15,16)$. Assim como Lasser et al. (15), acreditamos que nessa situação talvez a exenteração apresente um caráter meramente paliativo. Contudo, novos estudos com a adoção de novos agentes quimioterápicos e o uso da radioterapia intraoperatória possam quem sabe modificar este panorama atual.

A recidiva regional é um problema significativo mesmo após a cirurgia pélvica radical (2). O índice de recidiva no presente estudo foi alto de 48,1\% (13 pacientes). No entanto, quando foram analisadas somente as recidivas pélvicas, verificou-se que as mesmas ocorreram apenas no Grupo 1 (41,6\%). O Grupo 1 não apresentou recidiva local após o tratamento cirúrgico $(0 \%)$.

Esse fato pode estar relacionado à ausência de ressecções $\mathrm{R} 1$ neste grupo, haja vista que a exenteração é um dos principais fatores prognósticos de recidiva pélvica (1). Atualmente, o uso agressivo da terapia multimodal tem melhorado os resultados curativos nos pacientes com neoplasia retal avançada ou recidivada. Contudo, o tratamento neo-adjuvante em nosso estudo, não foi identificado como fator prognóstico importante na sobrevida.

Determinados estudos têm associado o uso de tratamento radio-quimioterápico neoadjuvante com a melhora dos índices de ressecção R0 nos tumores T4 ou nas recidivas pélvicas os quais não eram anteriormente considerados candidatos à cirurgia. Em estudo prévio realizado por Bedrosian et al. (7), 91\% dos pacientes submetidos a tratamento neo-adjuvante e cirurgia (exenteração ou não) culminaram com uma ressecção R0 em contraste com $71 \%$ dos pacientes não submetidos a tratamento neo-adjuvante $(\mathrm{P}=0,05)$. Paralelamente, houve uma tendência a maior sobrevida nos pacientes tratados, porém sem significância estatística $(\mathrm{P}=0,07)$. $\mathrm{O}$ efeito deste tratamento na sobrevida em longo prazo ainda necessita de comprovação (1). Embora exista estudo que comprove que a radioquimioterapia adjuvante pode melhorar a sobrevida nesse grupo de pacientes (17), sua morbidade é grande devido, principalmente, às aderências pós-operatórias, o que leva a ser considerado o tratamento neo- adjuvante como tendência da literatura $(18,19)$. Com o aumento do uso do tratamento radioterápico neoadjuvante, torna-se difícil o tratamento radioterápico adjuvante.

Em nosso estudo, apenas um paciente recebeu radio e quimioterapia no pós-operatório. Esse paciente pertencente ao Grupo 1, não havia recebido tratamento neo-adjuvante. Três pacientes do Grupo 2, por já terem recebido tratamento neo-adjuvante por ocasião da primeira cirurgia, não foram candidatos a um novo esquema de tratamento com radio-quimioterapia pré ou pós-operatória.

Vários autores tentaram descrever fatores prognósticos nos pacientes submetidos à exenteração pélvica por neoplasias de reto. Em nosso estudo, quando se fez a análise multivariada de fatores prognósticos, a variável independente para sobrevida foi à presença de linfonodos acometidos pela neoplasia $(\mathrm{P}=0,0167)$. Esse fato era esperado, haja vista que a profundidade de invasão da parede retal e a presença de linfonodos comprometidos são os principais indicadores prognósticos nesse tipo de tumor. Esses resultados foram de encontro aos já descritos por outros autores, onde a presença de linfonodos comprometidos foi o principal fator prognóstico negativo de sobrevida em longo prazo $(1,2)$. Assim como em outros estudos previamente descritos, no presente estudo foram considerados como fatores prognósticos negativos na análise univariada as seguintes variáveis: sexo masculino, comprometimento de linfonodos e tumor recidivado. A pelve feminina por razões meramente anatômicas pode colaborar com uma porcentagem maior de ressecções radicais (R0), o que pode explicar esse melhor prognóstico. Assim como os tumores primários que sabidamente apresentam melhores resultados com a ressecção multivisceral do que as recidivas $(1,2,9,10$, 15). Ao contrário de outros estudos, não identificamos a idade como fator significativo para sobrevida.

Meterissian et al. (17) relataram uma menor sobrevida em pacientes mais jovens, talvez devido à presença de fatores biológicos tumorais mais agressivos nesta população. Em contrapartida, Ike et al. (2), observaram que pacientes acima de 50 anos apresetavam pior prognóstico, principalmente devido às doenças associadas. Dessa maneira, mais estudos devem ser realizados para avaliar se a idade isoladamente pode ser definida como critério prognóstico.

É fato consumado que o grau de diferenciação do tumor pode influenciar a sobrevida, sendo que os 
Rev bras Coloproct Janeiro/Março, 2008
A Exenteração Pélvica para o Câncer de Reto: Avaliação dos Fatores Prognósticos de Sobrevida em 27 doentes Operados Sergio Renato Pais Costa e Cols.
Vol. 28 mais diferenciados têm maior probabilidade de sobrevida. Esse fato foi confirmado nesse estudo, onde os doentes com neoplasias pouco e moderadamente diferenciadas apresentaram menor probabilidade de sobrevida do que naqueles com neoplasias bem diferenciadas.

Nenhum dos pacientes com neoplasias pouco diferenciadas alcançou sobrevida além de 3 anos. Esse fato pode estar relacionado ao perfil mais agressivo destas neoplasias, as quais geralmente apresentam uma maior tendência à recidiva $(18,19)$.

Vários autores indicam a exenteração pélvica como tratamento paliativo em pacientes muito sintomáticos. Esta abordagem é controversa, haja vista que não proporciona um prolongamento da sobrevida, e acarreta morbidade e mortalidade significativas. Em casos extremamente selecionados ao nosso entender ela pode ser realizada, pois pode temporariamente paliar os sintomas severos que pioram a qualidade de vida dos doentes. Sintomas como fístulas reto-vaginais, retovesicais e hemorragia são de difícil controle clínico, o que pode justificar o seu tratamento cirúrgico, principalmente, em indivíduos selecionados (jovens com baixo risco cirúrgico-anestésico).
Além do caráter curativo, a exenteração pode melhorar os sintomas da doença. De vinte e um doentes que apresentavam sintomas graves na presente casuística, alguns incapacitantes houve uma remissão completa dos sintomas em 17 (80,9\%). Nos pacientes nos quais os sintomas não foram eliminados, esses foram ao menos temporariamente resolvidos, justificando também o procedimento.

\section{CONCLUSÃO}

A exenteração pélvica para o tratamento do câncer de reto localmente avançado (T4) e da recidiva pélvica exclusiva é uma operação de alta morbidade e considerável mortalidade em nosso meio. Os fatores prognósticos adversos identificados pela análise univariada foram: sexo masculino, comprometimento de linfonodo e tipo de tumor (recidiva pélvica). Contudo, na análise multivariada somente comprometimento linfonodal foi identificado como fator prognóstico negativo. Assim, essa formidável operação deve ser considerada com reservas, tendo seu melhor resultado em longo prazo nos doentes com tumores Estádio T4 sem comprometimento linfonodal.

ABSTRACT: Aim - To identify survival prognostic factors in patients who underwent curative pelvic exenteration from rectal adenocarcinoma (T4 tumors and isolated pelvic recurrence). Methods - Complete follow-up data were available on 27 patients who underwent this surgical procedure between 1996 and 2006. Multiple prognostic factors were studied by multivariate analysis (epidemiological, surgical and histological). Results - Postoperative mortality was $7 \%(\mathrm{n}=2)$ while overall morbidity was $55 \%$ $(n=15)$. Mean of overall survival was 38 months. $T 4$ tumors presented more long-time survival than pelvic recurrence (47 X 26 months). Lymph node disease $(\mathrm{N}+)$ was single adverse prognostic factor at multivariate analysis. Conclusion - Pelvic exenteration presents both high morbidity and considerable mortality. It should be reserved for $\mathbf{T} 4$ tumors, principally when there was no lymph node spreading.

Key words: Pelvic Exenteration, Rectal Cancer, Prognostic Factors, Morbidity, Mortality, Treatment Outcome.

\section{REFERÊNCIAS}

1. Ike H, Shimada H, Yamaguchi S, Ichikawa Y, Fujii S, Ohki S. Outcome of total pelvic exenteration for primary rectal cancer. Dis Colon Rectum 2001; 46: 474-480.

2. Yamada K, Ishizawa T, Niwa K, Chuman Y, Aikou T. Pelvic Exenteration and Sacral Resection for Locally Advanced Primary and Recurrent Rectal Cancer. Dis Colon Rectum 2002; 45 (8): 1078-84.

3. Akasu T, Yamaguchi T, Fujimoto Y, Ishiguro S, Yamamoto S, Fujita S, Moriya Y. Abdominal sacral resection for posterior pelvic recurrence of rectal carcinoma: analyses of prognostic factors and recurrence patterns. Ann Surg Oncol 2007; 41 (1): 74-83.

4. Sasson AR, Sigurdson ER. Management of locally advanced rectal cancer. Surg Oncol 2000; 9: 193-204.

5. Saito N, Koda K, Takiguchi N. Curative surgery for local pelvic recurrence of rectal cancer. Dig Surg 2003; 20 (3): 192200.

6. Caricato M, Borzomati D, Ausania F, Valeri S, Rosignoli A, Coppola R. Prognostic factors after surgery for locally recurrent rectal cancer: an overview. Eur J Surg Oncol 2006; 32(2): 126-32.

7. Bedrosian I, Giacco G, Penderson L, Rodrigues-Bigas MA, Feig B, Hunt KK et al. Outcome after curative resection for 
locally recurrent rectal cancer. Dis Colon Rectum 2006; 49(2): 175-82.

8. Kakuda JT, Lamont JP, Chu DZ, Paz IB. The role of pelvic exenteration in management of recurrent rectal cancer. Am J Surg 2003; 186 (6): 660-4.

9. Pawlik TM, Skibber JM, Rodriguez-Bigas MA. Pelvic Exenteration for Advanced Pelvic Malignancies. Ann Surg Oncol 2006; 13 (5): 612-23.

10. Moriya Y, Akasu T, Fujita S, Yamamoto S. Aggressive surgical treatment for patients with T4 rectal cancer. Colorectal Dis 2003; 5(5): 427-31.

11. Kecmanovic DM, Pavlov MJ, Kovacevic PA, Septkoviski AV, Ceranic MS, Stamenkovic AB. Management of advanced pelvic cancer by exenteration.Eur J Surg Oncol 2003; 29 (9): 743-6.

12. Lopez MJ, Luna-Pérez P. Composite pelvic exenteration: is it worthwhile? Ann Surg Oncol. 2004; 11(1):27-33.

13. Russo P, Ravindran B, Katz J, Paty P, Guillem J, Cohen AM. Urinary diversion after total pelvic exenteration for rectal cancer. Ann Surg Oncol. 1999; 6(8):732-8.

14. Puntambekar S, Kudchadkar RJ, Gurjar AM, Sathe RM, Chaudhari YC, Agarwal GA et al. Laparoscopic pelvic exenteration for advanced pelvic cancers: a review of 16 cases. Gynecol Oncol. 2006; 102(3):513-6.
15. Lasser P, Doidy L, Elias D, Lusinchi A, Sabourin JC, Bonvalot $S$, et al. Total pelvic exenteration and rectal cancer. Apropos of 20 cases. Chirurgie. 1999; 124(3):252-7.

16. Reerink O, Mulder NH, Botke G, Sluiter WJ, Szabó BG, Plukker JT et al. Treatment of locally recurrent rectal cancer, results and prognostic factors Eur J Surg Oncol. 2004; 30(9): 954-8.

17. Meterissian SH, Skibber JM, Giacco GG, el-Naggar AK, Hess KR, Rich TA. Pelvic exenteration for locally advanced rectal carcinoma: factors predicting improved survival.Surgery. 1997; 121(5): 479-87.

18. Costa F, Saad ED, Hoff PM. In: Buzaid AC, Hoff PM. Manual Prático de Oncologia Clínica. 2006, São Paulo. Ed. Dêndrix: 93-8.

19. Chang GJ, Feig BW. In: Feig BW, Berger DH, Fuhrman GM. The M.D. Anderson Surgical Oncology Handbook. 2006, Philadelphia. Lippincot-Williams \& Wilkins: 261-319.

\section{Endereço para correspondência:}

Instituto de Oncologia São Paulo - Sergio Renato Pais Costa Av. Pacaembu, 1400

0123-200

Email:sergiorenatopais@ig.com.br Tel: 11- 3666-2299 